\title{
Transient shear banding in time-dependent fluids
}

\author{
Xavier Illa, ${ }^{1,2}$ Antti Puisto, ${ }^{2}$ Arttu Lehtinen, ${ }^{2}$ Mikael Mohtaschemi, ${ }^{2}$ and Mikko J. Alava ${ }^{2}$ \\ ${ }^{1}$ Departament d'Estructura i Constituents de la Matèria, Facultat de Física, Universitat de Barcelona, Diagonal, \\ 647, E-08028 Barcelona, Catalonia, Spain \\ ${ }^{2}$ Aalto University, School of Science, Department of Applied Physics, P.O. Box 14100, FI-00076 AALTO, Finland
}

(Received 27 September 2012; revised manuscript received 4 January 2013; published 22 February 2013)

\begin{abstract}
We study the dynamics of shear-band formation and evolution using a simple rheological model. The description couples the local structure and viscosity to the applied shear stress. We consider in detail the Couette geometry, where the model is solved iteratively with the Navier-Stokes equation to obtain the time evolution of the local velocity and viscosity fields. It is found that the underlying reason for dynamic effects is the nonhomogeneous shear distribution, which is amplified due to a positive feedback between the flow field and the viscosity response of the shear thinning fluid. This offers a simple explanation for the recent observations of transient shear banding in time-dependent fluids. Extensions to more complicated rheological systems are considered.
\end{abstract}

DOI: 10.1103/PhysRevE.87.022307

PACS number(s): 83.50.Ax, 83.60.Rs, 83.60.Wc

The property that characterizes complex fluids is their nontrivial rheology, shear rate-stress relation. They are generally further categorized into shear thinning or shear thickening fluids. Both cases are additionally complicated by time dependence. Due to the stress-shear interaction, already small perturbations in the local stress can result in a positive feedback with the flow promoting shear instabilities in each case $[1,2]$. The understanding of complex fluids is of enormous importance for many practical applications [3] and the theory touches on many branches of physics. Recent advances make it possible to follow the suspension local velocity during a standard rheological experiment $[4,5]$. Quantifying the local flow field simultaneously with rheological measurements gives the possibility to measure both the intrinsic and apparent rheology. This has led to the discovery that a heterogeneous shear distribution in samples during such tests is ubiquitous. Shear banding [6] has been observed in many systems composed of substantially different building blocks, such as colloidal glasses, wormlike micelles, foams, and granular matter [7]. The current viewpoint, both phenomenologically and theoretically, is that a nonmonotonic intrinsic flow curve is what is common to most of these materials $[6,8]$, but also other mechanisms have been suggested [9].

A branch of complex fluids are the simple yield stress fluids [10]. These materials do not show aging phenomena (thixotropy). Therefore, they are expected to have a monotonic intrinsic flow curve and a steady state without shear bands [11]. However, recent experiments [12] display shear banding during startup flows in a rotational rheometer indicating timedependent behavior. These so called transient shear bands can be very long lasting, but eventually vanish with a homogeneous steady state. The transient shear banding phenomenon tests our fundamental understanding of non-Newtonian fluids, and is also important for industrial processes and simply for understanding usual rheological measurements. A particular feature of the transient shear banding is that it appears to exhibit scaling familiar from critical phenomena: The time it takes for the transient to disappear (fluidization time $\tau_{f}$ ) is a power-law function of the shear rate or applied stress [12].

For the reasons leading to transient shear banding (see the summary by Adams et al. [13]), three main candidates are offered. (i) With certain parameters, the dynamical equations are unstable amplifying small perturbations, which slowly quench towards the homogeneous steady state [14]. (ii) The fluid flow curve is time dependent and can, at different times, have nonmonotonic shape [15]. (iii) Elastic stress overshoots cause instability in the flow [16]. Some theoretical models appear to produce transient shear banding including shear transformation zone theories [17], a modified soft glassy rheology model [18], a simplified fluidity model [18], and a mesoscopic model of plasticity [19]. Such models of transient shear banding share the property of time-dependent reduction of the local stress under shear [17-19], as explained in Ref. [20]. The literature reports experimental evidence of time-dependent rheology in carbopol gels [12], especially at small shear rates, as well as in other simple yield stress fluids [21], appearing as slight hysteresis in the flow curves. Further details of the time-dependent flow curve hysteresis related to carbopol gels is reported in Ref. [22]. Stronger hysteresis is inherent to thixotropic fluids [21]. Indeed, transient shear banding has been recently found also there, well above the critical shear rates [23], indicating that transient shear banding could be a general property of soft glassy materials.

Motivated by these findings, we consider here a structural model to find the main ingredients of transient shear banding. It is for a simple time-dependent Newtonian fluid, purposely neglecting other complications present in yield stress fluids and thixotropic fluids, such as elastic and yield stresses and critical shear rates. Spatial resolution, necessary for the transient shear banding, is obtained when the rheological model is coupled to the Navier-Stokes equation for laminar flow in a concentric cylinder Couette device. The transient shear banding here occurs due to the shear rate-viscosity coupling in the Navier-Stokes solution. We demonstrate that such transients initiate since the shear stress exhibits a finite gradient in any rotational geometry. This gradient is amplified by the interaction with the shear thinning fluid. In the model, the time scales associated with the relaxation of the structure depend on the shear rate. A higher shear rate implies faster dynamics, creating, with the coupled Navier-Stokes equations, an amplifying effect during the startup flow. 
Model. Most microscopic descriptions of the structure of a complex fluid are purely phenomenological and based on a kinetic relaxation equation. The simplest $\lambda$ models stipulate an evolution equation for a structural parameter, $\lambda[4,24]$. This describes the internal order, such as the state of aggregation in colloids or the alignment of particles with shear [4]. Usually, shear and/or temperature influences the temporal evolution of $\lambda$ [24]. The macroscopic rheology is obtained coupling $\lambda$ to a constitutive equation. Variants of $\lambda$ models can be used to describe the flow curves of thixotropic, simple yield stress fluids, and shear thinning fluids $[4,25]$. They generally assume homogeneous flow and thus are not applicable to shear bands.

In aggregating suspensions and microgels a portion of the liquid is trapped due to the presence of solid structures [26,27]. This is, in fact, also the interpretation shared in the literature for carbopol microstructure, which is observed to be formed of elastic spongelike elements [27,28]. Therefore, instead of using an abstract structure parameter (with no direct physical interpretation) it is more sensible to select the immobilized volume fraction $\phi$, which describes the jammed fluid, as has been done in approaches using the population balance equations [26]. The simplest time-dependent rheology follows by including a temporal relaxation of the volume fraction to a steady state. Unlike the relaxation rate, the steady state volume fraction is independent of the applied shear rate corresponding to a Newtonian fluid. Such a model can be considered a Taylor expansion of the dynamical equation for the volume around the steady state. The resulting kinetic equation is given,

$$
\frac{d \phi}{d t}=\frac{A_{b}}{\left(\mu / \mu_{o}\right)^{m}}+\left(A_{s}-B_{s} \phi\right)\left(\frac{\dot{\gamma}}{\dot{\gamma}_{0}}\right)^{k}
$$

where $A_{s}\left(B_{s}\right)$ is the kinetic constant for the shear growth (destruction), $\dot{\gamma}$ is the magnitude of the shear rate, and $k$ and $\dot{\gamma}_{0}$ (set to unity) both relate to the volume fraction sensitivity to shearing. $A_{b}, \mu_{o}$, and $m$ describe the growth of jammed volume fraction due to the shear independent motion of the structure elements. The special case of $m=k$ presents a simple yield stress fluid, $m>k$ gives a nonmonotonic flow curve indicating thixotropy, and $m<k$ produces shear thinning behavior. In what follows, we fix the parameters $A_{s}$ and $B_{s}$ to 0.665 and 1.0 , respectively. Since we are concentrating on a minimum model showing transient shear banding, in the following we set $A_{b}=0$ and parametrize the initial volume fraction instead of specifying the sample history and the associated parameters. It is well known that in any practical experiment the initial state depends on the sample history and the shear independent structure dynamics. Therefore, special attention has to be paid to the measuring protocol. The shear independent terms dominate the structure evolution at small shear rates, making the flow curve non-Newtonian, but have negligible influence in the transient shear banding regime. There it is reasonable to assume that both the structure growth and the destruction terms are determined by the shear rate, justifying such approximation. The exact form of the rate kernels are presently unclear and here we choose a simple power-law dependence. The scaling factors $\dot{\gamma}_{0}$ and $\mu_{o}$ make the equation unitless, and could be incorporated to the kernels $A_{s}, A_{b}$, and $B_{s}$ equally well.
We use the well-known Krieger-Dougherty constitutive equation [29], which reads

$$
\mu(\phi)=\mu_{0}\left(1-\frac{\phi}{\phi_{m}}\right)^{-\eta},
$$

where $\phi_{m}$ is the jamming volume fraction, $\mu_{0}$ the liquid viscosity, and $\eta$ gives the scaling of the viscosity. From now on these quantities are set to $\phi_{m}=0.68$ (random sphere packing), $\mu_{0}=1 \mathrm{mPas}$ (water), and $\eta=1.82$ [30]. These values relate to the initial conditions at which the transient shear banding appears, but are irrelevant, e.g., for the scaling relations of the fluidization times.

The steady state volume fraction is $\phi_{s s}=\frac{A_{s}}{B_{s}}$. As a consequence of leaving the shear independent terms out of the kinetic equation, the resulting steady state volume fraction is independent of the shear rate. As mentioned before, these shear independent processes are the ones that drive the system out of steady state implied by Eq. (1) when not sheared. This effect is incorporated into the model by simply initializing the system to the desired volume fraction before starting the structure evolution. For shear thinning fluids having no yield stress, the present approximation is valid for all shear rates. Fluids having a yield stress are properly described in the range where shear localization does not appear, i.e., the fluidization is complete. Besides fixing the steady state, $A_{s}$ and $B_{s}$ determine the rate of relaxation. Plugging $\phi_{s s}$ to the constitutive equation and applying the Newtonian assumption $\sigma_{s s}=\mu\left(\phi_{s s}\right) \dot{\gamma}$ gives the Newtonian steady state flow curve in Fig. 1. The stress shows time and shear rate dependent exponential relaxation (the inset), when started from $\phi_{o} \neq \phi_{s s}$, since the structure evolution follows Eq. (1).

For the spatial resolution we couple the model with a continuum description of the fluid flow in a Couette rheometer geometry [31]. The incompressible Navier-Stokes equation for laminar flow in that has the analytical one-dimensional (radial) solution [32]

$$
\dot{\gamma}(r)=\frac{\Omega_{b}-\Omega_{a}}{\left[\int_{R_{a}}^{R_{b}} \frac{1}{r^{3} \mu(r)} d r\right]} \cdot \frac{1}{r^{2} \mu(r)},
$$

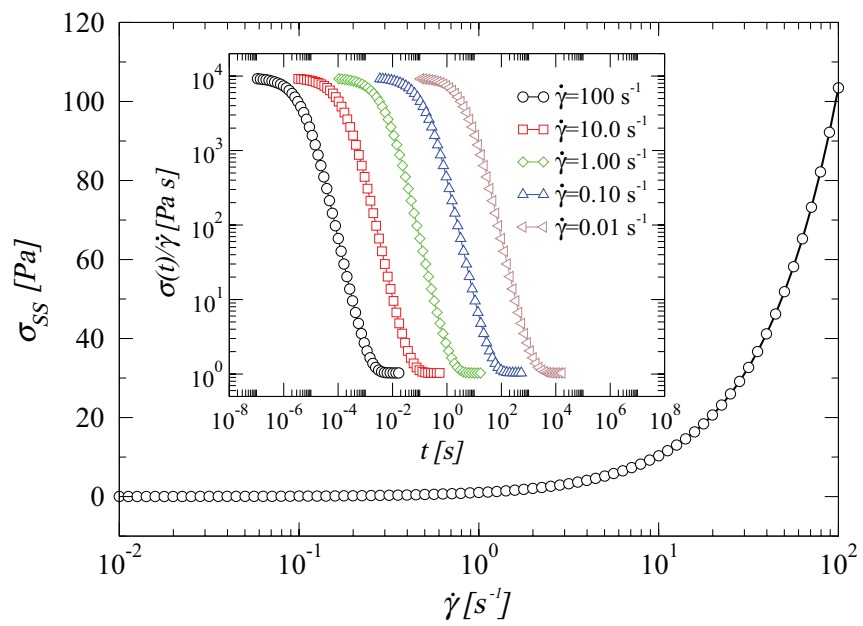

FIG. 1. (Color online) Steady state flow curve, showing Newtonian response, with $A_{s}=0.665, B_{s}=1.000$. (Inset) The transient behavior of the viscosity for $\phi_{0}=0.6799$, and $k=1.5$. 
where $r$ is the radial distance from the cell center, $\dot{\gamma}(r)$ is the local shear rate, $\Omega_{a}\left(\Omega_{b}\right)$ is the angular velocity of the inner (outer) cylinder, and $R_{a}\left(R_{b}\right)$ is the radius of the inner (outer) cylinder. Equation (3) implies that only the relative angular velocity of the two cylinders matters. In order to mimic the experiments of Ref. [12] only the inner cylinder is rotated and the radii are set to $R_{a}=23.9 \mathrm{~mm}$ and $R_{b}=25 \mathrm{~mm}$. Equation (3) implies a spatial dependence for the volume fraction $\phi(t, r)$. To this end, a radial discretization is applied with a uniform grid with $N$ sampling points, at each of which a separate $\phi$ is evolved. Thus, the time evolution of the local flow field is obtained when Eqs. (1) and (3) are iteratively solved using a forward Euler algorithm under a constant global shear rate. Solving the structure and flow field evolutions this way assumes that the time scales of the inertial effects and the structure kinetics are very different. The flow field quickly adapts the changes caused by the slowly varying time-dependent viscosity.

The global shear rate is defined as the radial average shear rate $\int_{R_{a}}^{R_{b}} \dot{\gamma}(r) /\left(R_{b}-R_{a}\right)$ for a Newtonian fluid in the Couette geometry. This reads

$$
\langle\dot{\gamma}\rangle=\left(\Omega_{b}-\Omega_{a}\right) \frac{2 R_{a} R_{b}}{R_{b}^{2}-R_{a}^{2}} .
$$

Other definitions for the "engineering" shear rate are also used $[33,34]$. All such are linearly proportional to the difference of the angular velocities, $\Omega_{b}-\Omega_{a}$.

Results. Varying the initial volume fraction and the kinetic exponent $k$ uncovers three different startup flow scenarios, as illustrated in the schematic phase diagram shown in Fig. 2. An almost homogeneous relaxation of the volume fraction profile arises at initial volume fractions above the steady state value and low kinetic exponents [Fig. 2(a)]. Increasing either makes the transient shear bands appear [Fig. 2(b)]. At initial volume fractions below the steady state, there is no transient shear bands [Fig. 2(c)]. The boundaries of that phase (gray area) are qualitative, as the transition from the homogeneous

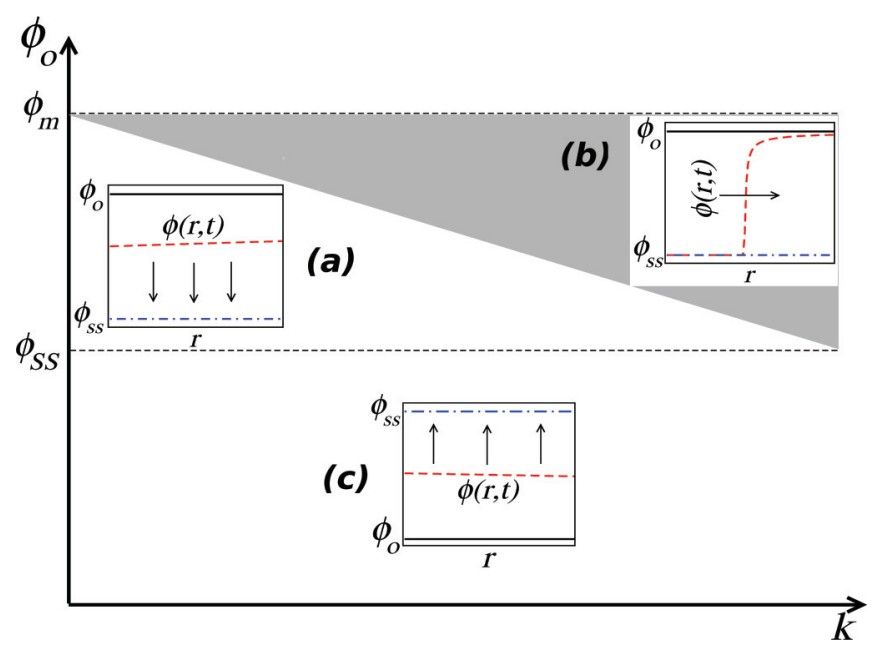

FIG. 2. (Color online) Schematic phase diagram of the instability/stability regimes as a function of $k$ and $\phi_{0}$. In regions (a) and (c) spatially homogeneous relaxation is observed, whereas region (b) exhibits transient shear banding.
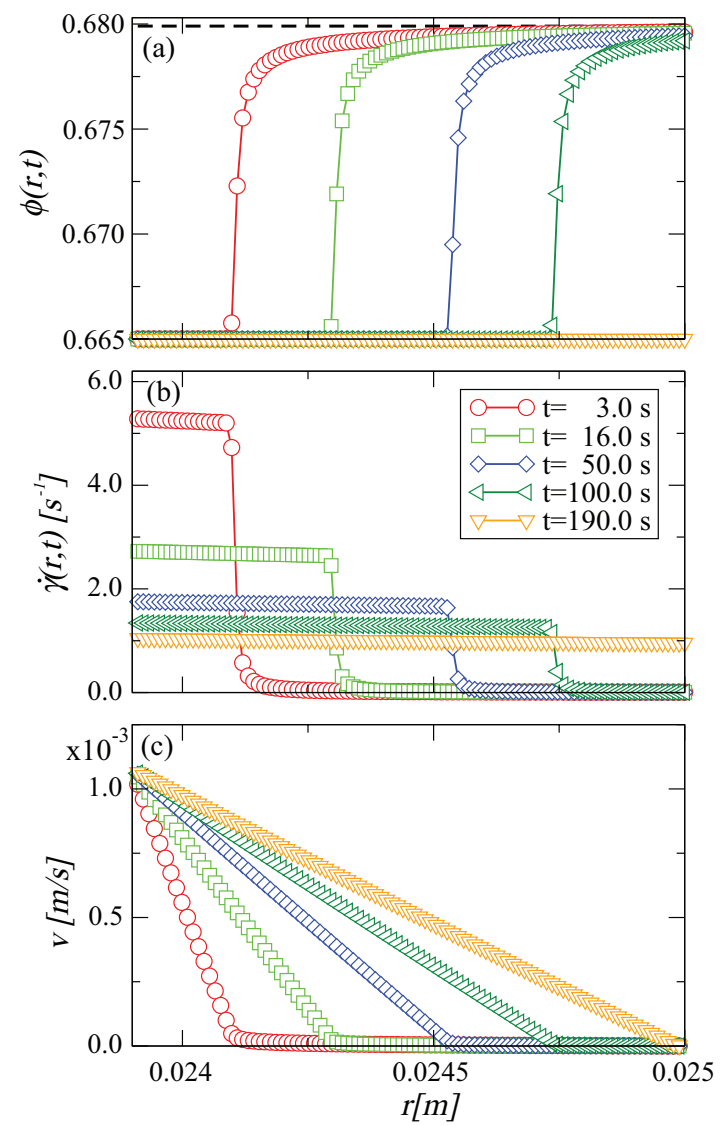

FIG. 3. (Color online) Volume fraction (a), shear rate (b), and velocity (c) profiles during the startup flow for $A_{s}=0.665, B_{s}=$ $1.000, k=1.5,\langle\dot{\gamma}\rangle=1.0 \mathrm{~s}^{-1}$ and $\phi(r, t=0)=0.6799$.

relaxation Fig. 2(a) to the transient shear banding Fig. 2(b) occurs smoothly.

For a given value of $k$, the initial value $\phi_{o}$ is chosen in order to be in the transient shear banding region (gray area in Fig. 2). Thus, the simulation starts close to jamming, at $\phi_{o}=\phi(r, t=0) \sim \phi_{m}$. Figure 3(b) shows the local shear rates corresponding to the evolution of the volume fraction plotted in Fig. 3(a), from the numerical solution of Eqs. (2) and (3). The shear rate profiles show the development of a transient shear bands having two clearly distinct bands evolving towards a homogeneous flow. Comparing the velocity profiles [Fig. 3(c)] with the carpobol gel experiments [12] shows close similarities, even if the model here exhibits no yield stress.

The origin of transient shear banding is a self-feeding mechanism, which can be understood considering Eqs. (1)-(3). When the fluid starts to evolve at a homogeneous high volume fraction, the $\phi(r)$ decreases fastest at the regions where $\dot{\gamma}(r)$ has the largest value [Eq. (1)]: close to the inner cylinder of the device. The accelerated decrease of $\mu(r)$ [Eq. (2)] due to the faster relaxation rate further increases the $\dot{\gamma}(r)$ [Eq. (3)] at the same location. This accelerates the decrease of the $\phi(r)$ [Eq. (1)] at the same position, creating a self-amplifying mechanism for the growth of transient shear banding. Since the steady state viscosity is constant, the $\phi(r)$ will decrease elsewhere in the device, only with a slower rate due to lower $\dot{\gamma}(r)$. Finally a homogeneous steady state profile is reached. 


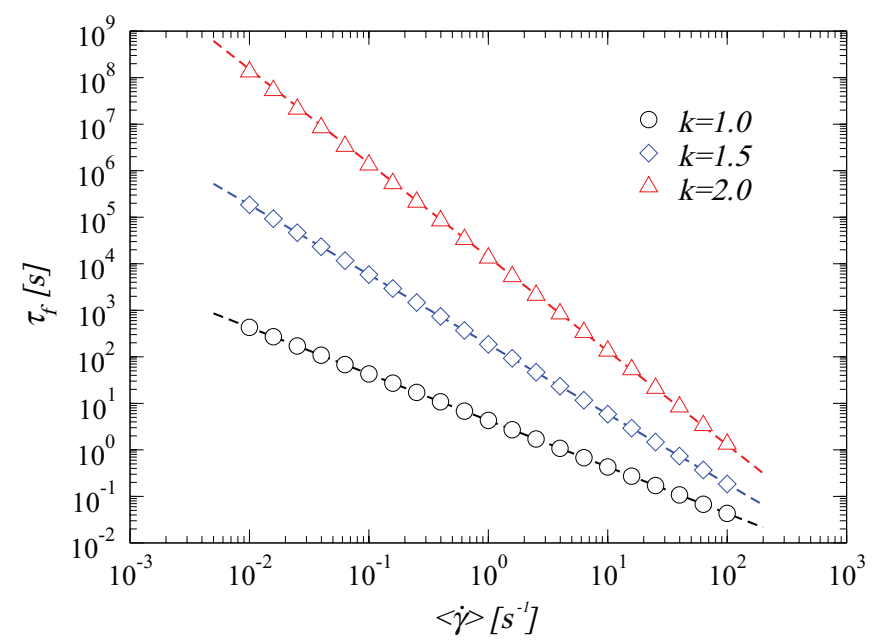

FIG. 4. (Color online) Fluidization times as a function of $\langle\dot{\gamma}\rangle$, for different values of $k$.

If the feedback either from $\phi(r)$ to $\dot{\gamma}(r)$ [Eqs. (2) and (3)] or from $\dot{\gamma}(r)$ to $\phi(r)$ [Eq. (1)] is not strong enough, the transient shear banding does not appear. The intensity of the feedback is adjusted by the exponent $k$ and the derivative of the Eq. (2) at the corresponding $\phi(r)$. If $\phi(r)$ at startup is below the steady state one, such a feedback loop does not exist; the growth of the $\phi(r)$ is fastest at the high shear rates, thus promoting the increase of the $\mu(r)$ and the decrease of the $\dot{\gamma}(r)$ at high shear rate regions. This reduces the shear rate differences in the gap.

It has been observed experimentally that in simple yield stress fluids the fluidization time decays with the global shear rate following a power law. Similar behavior is observed here (Fig. 4).

Here, the power-law decay follows $\tau_{f} \sim\langle\dot{\gamma}\rangle^{-k}$, with $k$ fixed by/to the exponent $k$ in Eq. (1). The other parameters or even the form of the viscosity function [Eq. (2)] do not influence the power-law slope. The rest of the model parameters and the gap width simply change the vertical position of the resulting power laws.

The combination of Eq. (1) with $A_{b}=0$ and Eq. (3) that describes the fluid in a Couette can be rewritten as

$$
\left(\frac{\dot{\gamma}_{0}}{\langle\dot{\gamma}\rangle}\right)^{k} \frac{d \phi(r, t)}{d t}=\left(A_{s}-B_{s} \phi(r, t)\right)[F(r, t)]^{k},
$$

where $[F(r, t)]^{k}$ represents the geometry effects and in particular is crucial for creating the transient shear bands. This form reveals a natural way to rescale the time: $t\langle\dot{\gamma}\rangle^{k}$. The measures dependent on the angular velocity can be rescaled with the global shear rate.

Other ways to illustrate transient shear banding, besides the velocity profiles, are to plot the temporal evolution of the local shear rate at the gap edges and the bandwidth [12]. These quantities are plotted with the same rescaling of time and shear rate in Figs. 5(a) and 5(b), respectively. The band edge is estimated as the position where $\left.\frac{\partial^{2} \hat{\gamma}(r, t)}{\partial r^{2}}\right|_{\delta}=0$. Figure 5 shows that there is a short induction period at small times, during which the shear rate localizes near the rotor. During the relaxation period, the shear rate decreases towards the steady state value as the transient shear bands vanish.

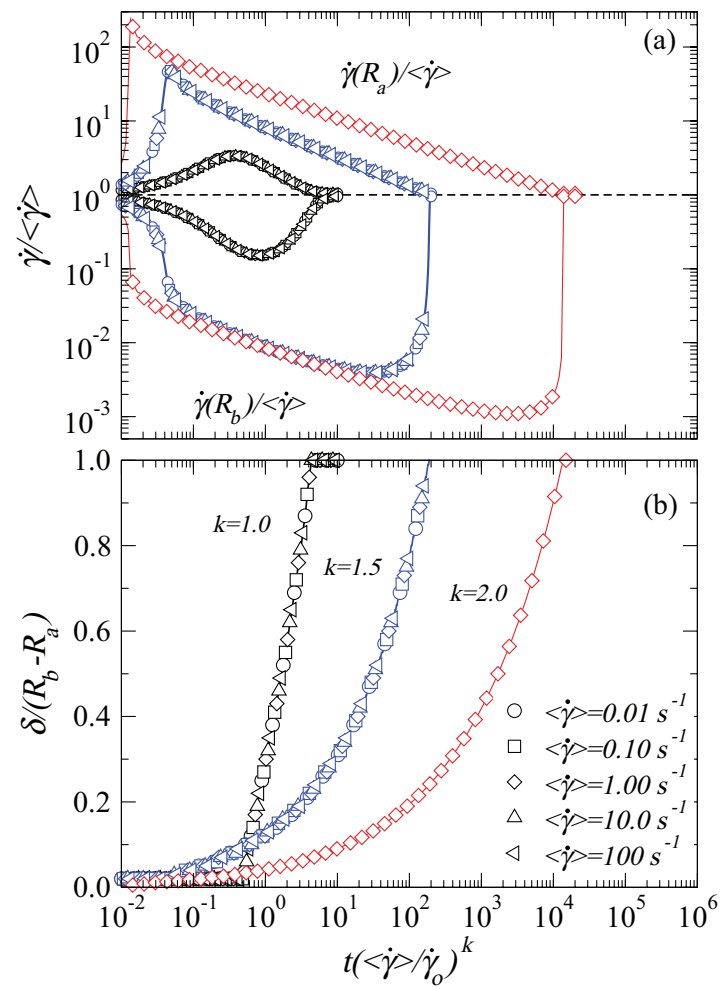

FIG. 5. (Color online) Normalized shear rate evolution close to the inner and outer cylinder (a), and the normalized evolution of the shear band edge position in the gap (b).

Divoux et al. [22] demonstrated that the fluidization time exponents in stress and shear rate controlled experiments relate to the steady state power-law exponent. Here, this equals unity. As one would expect, the fluidization for the stress controlled case follows a power law, $t_{f} \sim \sigma^{-k}$, as does the shear rate controlled one, $t_{f} \sim\langle\dot{\gamma}\rangle^{-k}$. A structural kinetics model producing a Herschel-Bulkley flow curve with a control of the exponent can be speculated to change also the the fluidization exponents towards the experimental ones [22]. To the best of our knowledge, such a model does not exist. Studying the properties of more advanced structural kinetics equations on the fluidization is therefore left to future studies.

Conclusions. We have studied transient shear banding, or fluidization of a simple time-dependent fluid. We constructed a minimal model which lacks further complications such as the (visco)elasticity of the structure, a physically motivated yield stress, the normal stresses suggested very recently [35] to play a role in steady state shear banding [25], or elastic stress overshoots [13]. Our analysis indicates that such conditions are not required for transient shear banding. On the contrary, transient shear banding should be a general feature of complex fluids like colloidal suspensions and microgels.

The transient shear bands during the fluidization were found to originate from the initial shear inhomogeneity here arising from the Couette geometry, which is amplified by the positive feedback coming from the shear dependent relaxation rates of the fluid. This mechanism should be present in all similar scenarios of fluidization: Studying the couplings built into the model implies that the same effect should occur in all practical measuring geometries for shear thinning complex 
fluids. This is since even the smallest stress gradient is enough to trigger the transient shear bands due to the shear dependent response of the fluid. Experimental observations of stress signatures associated with transient shear banding found in a cone-and-plate geometry, where the stress gradient is extremely small, also support this finding [36]. Furthermore, in Eq. (3) we neglected the inertial terms in the Navier-Stokes equation, which could play a role in the startup flow in such situations (referring to cone and plate). The self-amplifying mechanism between the flow and the fluid structure would be the same, but the origin of the shear inhomogeneity would come from the inertial terms rather than the flow geometry, which was the case in the circular Couette studied here.

The kinetic exponent related to the dependence of $\phi$ on $\dot{\gamma}$, is connected to the fluidization exponent in both the stress and the shear controlled cases. This indicates that studying the fluidization experimentally gives detailed information of the time scales of the internal relaxation processes in time-dependent fluids, which could be utilized to build proper structural models based on the experimental fluidization data. Such models could be established, for instance, around rheological models describing the volume fraction using population balances, which take into account the particle size distribution and concentration as rheological parameters [26]. Presently, work is devoted along these lines of research.

Acknowledgments. This work has been supported by the Effnet program in the Finnish Forest Cluster Ltd, and EU Framework 7 program SUNPAP. Also, the support from the Academy of Finland through the COMP center of excellence and the project number 140268 and within the framework of the International Doctoral Programme in Bioproducts Technology (PaPSaT) are acknowledged. The authors are grateful to S. Manneville and T. Divoux for fruitful discussions and a careful reading of the manuscript.
[1] D. A. Head, A. Ajdari, and M. E. Cates, Europhys. Lett. 57, 120 (2002).

[2] S. M. Fielding and P. D. Olmsted, Phys. Rev. Lett. 92, 084502 (2004).

[3] D. B. Braun and M. R. Rosen, Rheology Modifiers HandbookPractical Use and Application (William Andrew, Norwich, NY, 1999), first edition ed.

[4] D. Bonn, S. Rodts, M. Groenink, S. Rafa, N. Shahidzadeh-Bonn, and P. Coussot, Annu. Rev. Fluid Mech. 40, 209 (2008).

[5] S. Manneville, Rheol. Acta 47, 301 (2008).

[6] G. Ovarlez, S. Rodts, X. Chateau, and P. Coussot, Rheol. Acta 48, 831 (2009).

[7] P. Schall and M. van Hecke, Annu. Rev. Fluid Mech. 42, 67 (2010).

[8] G. Porte, J.-F. Berret, and J. L. Harden, J. Phys. II 7, 459 (1997).

[9] R. Besseling, L. Isa, P. Ballesta, G. Petekidis, M. E. Cates, and W. C. K. Poon, Phys. Rev. Lett. 105, 268301 (2010).

[10] A. Ragouilliaux, G. Ovarlez, N. Shahidzadeh-Bonn, B. Herzhaft, T. Palermo, and P. Coussot, Phys. Rev. E 76, 051408 (2007).

[11] A. Fall, J. Paredes, and D. Bonn, Phys. Rev. Lett. 105, 225502 (2010).

[12] T. Divoux, D. Tamarii, C. Barentin, and S. Manneville, Phys. Rev. Lett. 104, 208301 (2010).

[13] J. M. Adams, S. M. Fielding, and P. D. Olmsted, J. Rheol. 55, 1007 (2011).

[14] S. M. Fielding and P. D. Olmsted, Phys. Rev. E 68, 036313 (2003).

[15] K. A. Hayes, M. R. Buckley, H. Qi, I. Cohen, and L. A. Archer, Macromolecules (Washington, DC, US) 43, 4412 (2010).

[16] G. Marrucci and N. Grizzuti, J. Rheol. 27, 433 (1983).

[17] M. L. Manning, E. G. Daub, J. S. Langer, and J. M. Carlson, Phys. Rev. E 79, 016110 (2009).
[18] R. L. Moorcroft, M. E. Cates, and S. M. Fielding, Phys. Rev, Lett. 106, 055502 (2011).

[19] E. A. Jagla, J. Stat. Mech.: Theory Exp. (2010) P06006.

[20] R. L. Moorcroft and S. M. Fielding, Phys. Rev. Lett. (accepted), arXiv:1201.6259.

[21] P. Møller, A. Fall, V. Chikkadi, D. Derks, and D. Bonn, Phil. Trans. R. Soc. A 367, 5139 (2009).

[22] T. Divoux, C. Barentin, and S. Manneville, Soft Matter 7, 8409 (2011).

[23] J. D. Martin and T. Hu, Soft Matter 8, 6940 (2012).

[24] J. Mewis and N. Wagner, Adv. Colloid Interface Sci. 147-148, 214-227 (2009).

[25] P. C. F. Møller, J. Mewis, and D. Bonn, Soft Matter 2, 274 (2006).

[26] A. R. Heath, P. A. Bahri, P. D. Fawell, and J. B. Farrow, AIChE J. 52, 1641 (2006).

[27] J. M. Piau, J. Non-Newtonian Fluid Mech. 144, 1 (2007).

[28] F. K. Oppong, L. Rubatat, A. E. Bailey, B. J. Frisken, and J. R. de Bruyn, Phys. Rev. E 73, 041405 (2006).

[29] I. Krieger and T. Dougherty, Trans. Soc. Rheol. 3, 137 (1959).

[30] D. Quemada, Appl. Rheol. 18, 53298 (2008).

[31] N. Roussel, R. L. Roy, and P. Coussot, J. Non-Newtonian Fluid Mech. 117, 85 (2004).

[32] R. J. Phillips, R. C. Armstrong, R. A. Brown, A. L. Graham, and J. R. Abbott, Phys. Fluids A 4, 30 (1992).

[33] P. Estellé, C. Lanos, and A. Perrot, J. Non-Newtonian Fluid Mech. 154, 31 (2008).

[34] L. Bécu, P. Grondin, A. Colin, and S. Manneville, Colloids Surf., A 263, 146 (2004).

[35] I. Cheddadi, P. Saramito, and F. Graner, J. Rheol. 56, 213 (2012).

[36] T. Divoux, D. Tamarii, C. Barentin, S. Teitel, and S. Manneville, Soft Matter 8, 4151 (2012). 